\title{
Elevation of Memory Cytotoxic T Lymphocytes, Including Human T Lymphotropic Virus Type 1 Tax-Specific and Hepatitis Virus Type C-Specific Cytotoxic T Lymphocytes, in a Patient with Adult T-Cell Leukemia/Lymphoma and Hepatocellular Carcinoma
}

\author{
Tatsuro Jo $^{a}$ Yohei Kaneko $^{b}$ Takayuki Oishic Kaori Matsuzakab Haruna Shioyab \\ Takahiro Sakai $^{\mathrm{b}}$ Hiroo Tomoinagab Shizuka Hayashib ${ }^{\mathrm{b}}$ Masatoshi Matsuo ${ }^{\mathrm{a}}$ \\ Jun Taguchia \\ aDepartment of Hematology, Japanese Red Cross Nagasaki Genbaku Hospital, Nagasaki, Japan; \\ ${ }^{b}$ Department of Laboratory, Japanese Red Cross Nagasaki Genbaku Hospital, Nagasaki, Japan; \\ 'Department of Gastroenterology, Japanese Red Cross Nagasaki Genbaku Hospital, Nagasaki, Japan
}

Keywords

Adult T-cell leukemia/lymphoma · Cytotoxic T lymphocyte $\cdot$ Human T lymphotropic virus type 1 Tax $\cdot$ Hepatocellular carcinoma $\cdot$ Hepatitis virus type $C$

\begin{abstract}
Herein, we present the case of a patient who suffered from adult T-cell leukemia/lymphoma (ATLL) and hepatocellular carcinoma (HCC) after obtaining a sustained virological response following treatment with a direct-acting antiviral (DAA) at different points in time. The patient went into complete remission (CR) for ATLL. Unfortunately, subsequent relapse of ATLL was observed. This situation was overcome using chemotherapy with pegylated interferon alpha2b. Human T lymphotropic virus type 1 Tax-specific cytotoxic T lymphocytes (CTLs) were recognized after obtaining second $C R$, and those CTLs have been maintained for many years. After 4 years from the second $C R$, chronic hepatitis type $C$ was treated with a DAA, and sustained virological response was attained. However, the occurrence of HCC was detected. Surprisingly, the tumor disappeared spontaneously. Hepatitis virus type C-specific CTLs were also detected in the patient. T-cell receptor (TCR) $V$ beta gene repertoire analyses revealed oligoclonal expansion of effector and memory CTLs. The number of CTLs expressing the TCR $V$ beta 13.1 has increased over the years since HCC occurrence. The activation and maintenance of anticancer cellular immunity may have allowed the patient to obtain long-term survival and overcome two lethal neoplasms.




\section{Case Reports in Oncology}

\begin{tabular}{l|l}
\hline Case Rep Oncol 2020;13:802-806 \\
\hline DOI: 10.1159/000508092 & $\begin{array}{l}\text { @ 2020 The Author(s). Published by S. Karger AG, Basel } \\
\text { www.karger.com/cro }\end{array}$ \\
\hline
\end{tabular}

Jo et al.: Elevation of Tax-Specific and HCV-Specific CTLs in a Patient

\section{Introduction}

Host defense mechanisms against neoplasms comprise humoral and cellular immunity. Cytotoxic T lymphocytes (CTLs) are one of the major cell types that eliminate cancer cells. CTLs recognize tumor-specific antigens presented by the major histocompatibility complex of antigen-presenting cells [1]. Antigens of non-human origin may promote a stronger immune reaction than those of human origin. Herein we present a unique case involving a patient who suffered from adult T-cell leukemia/lymphoma (ATLL) and hepatocellular carcinoma (HCC) at two separate time points. The patient developed ATLL and obtained complete remission (CR) following chemotherapy and treatment with pegylated interferon alpha- $2 \mathrm{~b}$. However, the patient had HCC as the second malignancy several years later. Surprisingly, the HCC disappeared spontaneously. The immunological analyses revealed increased expression of effector and memory CTLs, including human T lymphotropic virus type 1 (HTLV-1) Tax-specific and HCV-specific CTLs, in the patient.

\section{Case Report/Case Presentation}

A 65-year-old female patient was diagnosed with acute type ATLL in January 2010. She was previously diagnosed with chronic hepatitis type $\mathrm{C}$ via thorough examinations, including liver biopsy, in 2006. Chemotherapy using multiple anticancer agents, called VCAP-AMP-

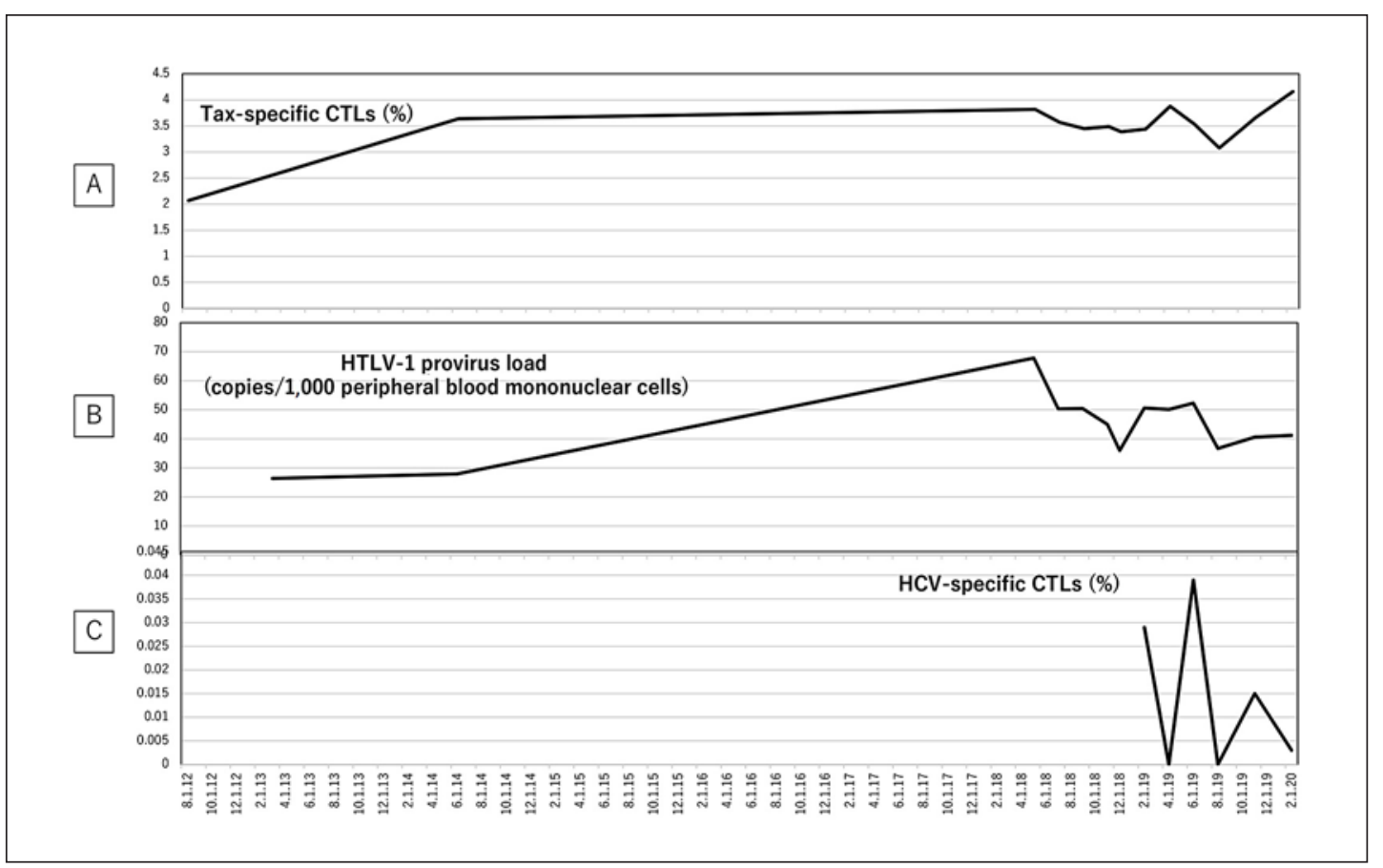

Fig. 1. The course of HTLV-1 Tax- and HCV-specific CTLs and HTLV-1 provirus load. A Tax-specific CTLs. The patient has human leukocyte antigen (HLA)-A*24:02. HLA-A*24:02 restricted Tax tetramer (HLA-A*24:02 HTLV-1 Tax 301--309 Peptide, MBL, Nagoya, Japan) was used for detecting Tax-specific CTLs. B HTLV-1 provirus load. The analyses were performed by SRL cooperation (Tokyo, Japan). C HCV-specific CTLs. HLAA*24:02 restricted HCV tetramer (HLA-A*24:02 HCV E2 717--725 Tetramer-EYVLLLFLL, MBL, Nagoya, Japan) was used for detecting HCV-specific CTLs.

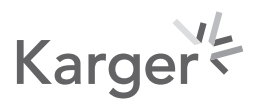




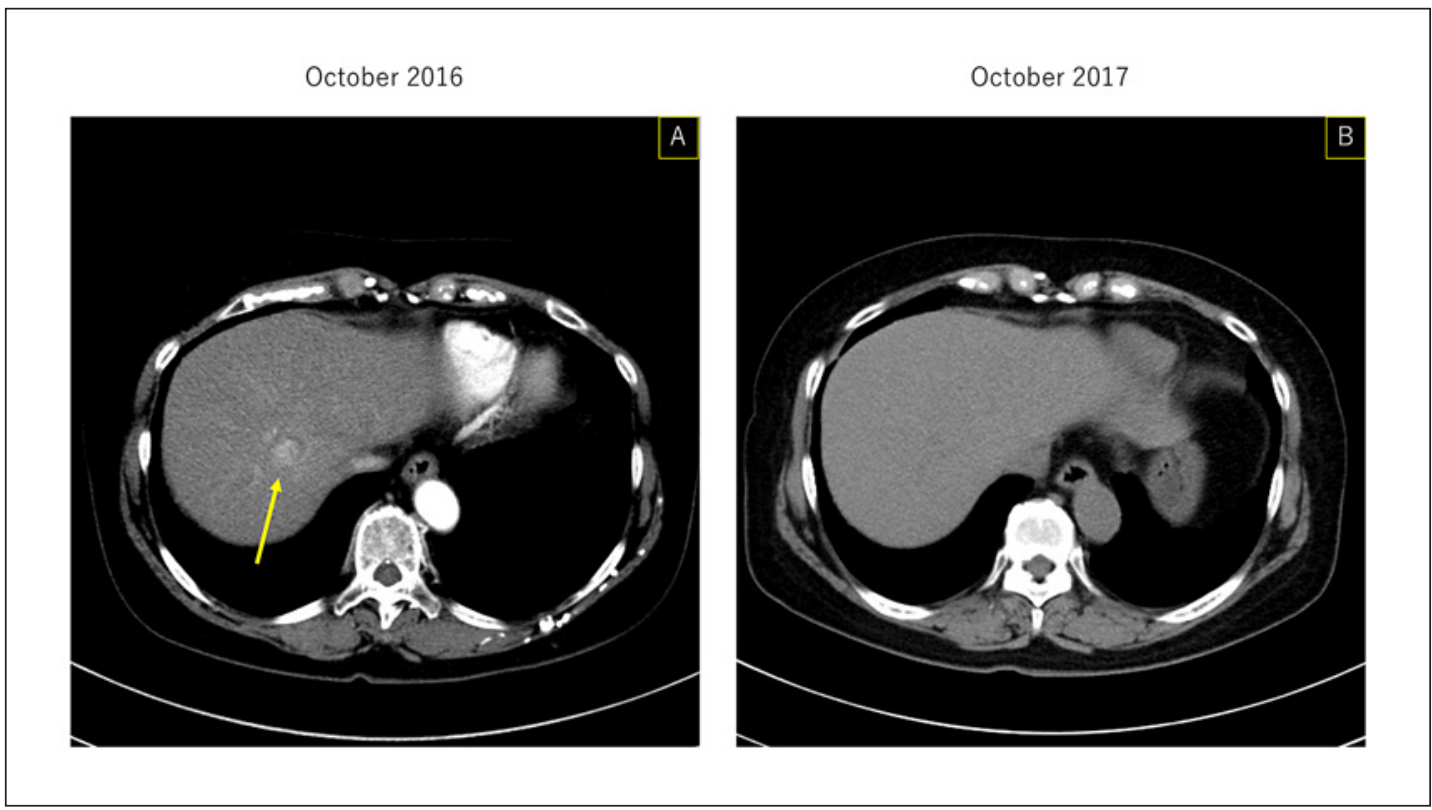

Fig. 2. Disappearance of hepatocellular carcinoma. A A solitary enhanced tumor of $20 \mathrm{~mm}$ in diameter in the segment VIII of the liver was detected in the enhanced CT analysis performed in October 2016. A yellow arrow indicates the tumor. B The tumor could not be detected in the plain CT analysis performed in October 2017.

VECD (mLSG15) therapy [2], was administered for six cycles and CR was obtained. However, relapse was identified in August 2010. The patient was administered six subsequent cycles of EPOCH therapy [3] until March 2011. Pegylated interferon alpha-2b was also used during each treatment cycle. The second CR was obtained and has been maintained to date. Tetramer analyses showed that Tax-specific CTLs have been maintained at high rates (>2\%) (Fig. 1A). However, the HTLV-1 virus was not eliminated. The HTLV-1 proviral load has been in the range of 25-65 copies/1,000 peripheral mononuclear cells after achieving the second CR (Fig. 1B).

The patient had been treated with sofosbuvir/ledipasvir, a combination of direct-acting antiviral (DAA) drugs, against chronic hepatitis type C for 12 weeks since December 2015. After treatment, HCV had not been detected since February 2016, indicating a sustained virological response. However, a solitary tumor of $20 \mathrm{~mm}$ in diameter was observed in the segment VIII region of the liver by ultrasound, computed tomography (CT) scan, and magnetic resonance imaging in October 2016 (Fig. 2A). Distant metastases were not detected. The patient was diagnosed with HCC, and an operational resection was planned. However, the tumor diameter decreased to $14 \mathrm{~mm}$ as evidenced by the CT scan performed in January 2017. Therefore, the operation was canceled, and a careful follow-up strategy was pursued. The CT scan analyses revealed that the tumor diameter further decreased to $10 \mathrm{~mm}$ in March 2017. Finally, no tumor was detected in October 2017 (Fig. 2B). The tumor was not detected by subsequent ultrasound analysis (data not shown). No relapse of HCC has been detected to date. HCV-specific CTLs have been monitored by tetramer analyses since February 2019 (Fig. 1C).

T-cell receptor (TCR) $V$ beta gene repertoire analyses were performed to analyze the clonality of the CD8+ effector and memory CTLs before (January 2015) and after $(2018,2019$, and 2020) the occurrence of HCC (Fig. 3). Oligoclonal expansion of effector and memory CTLs 


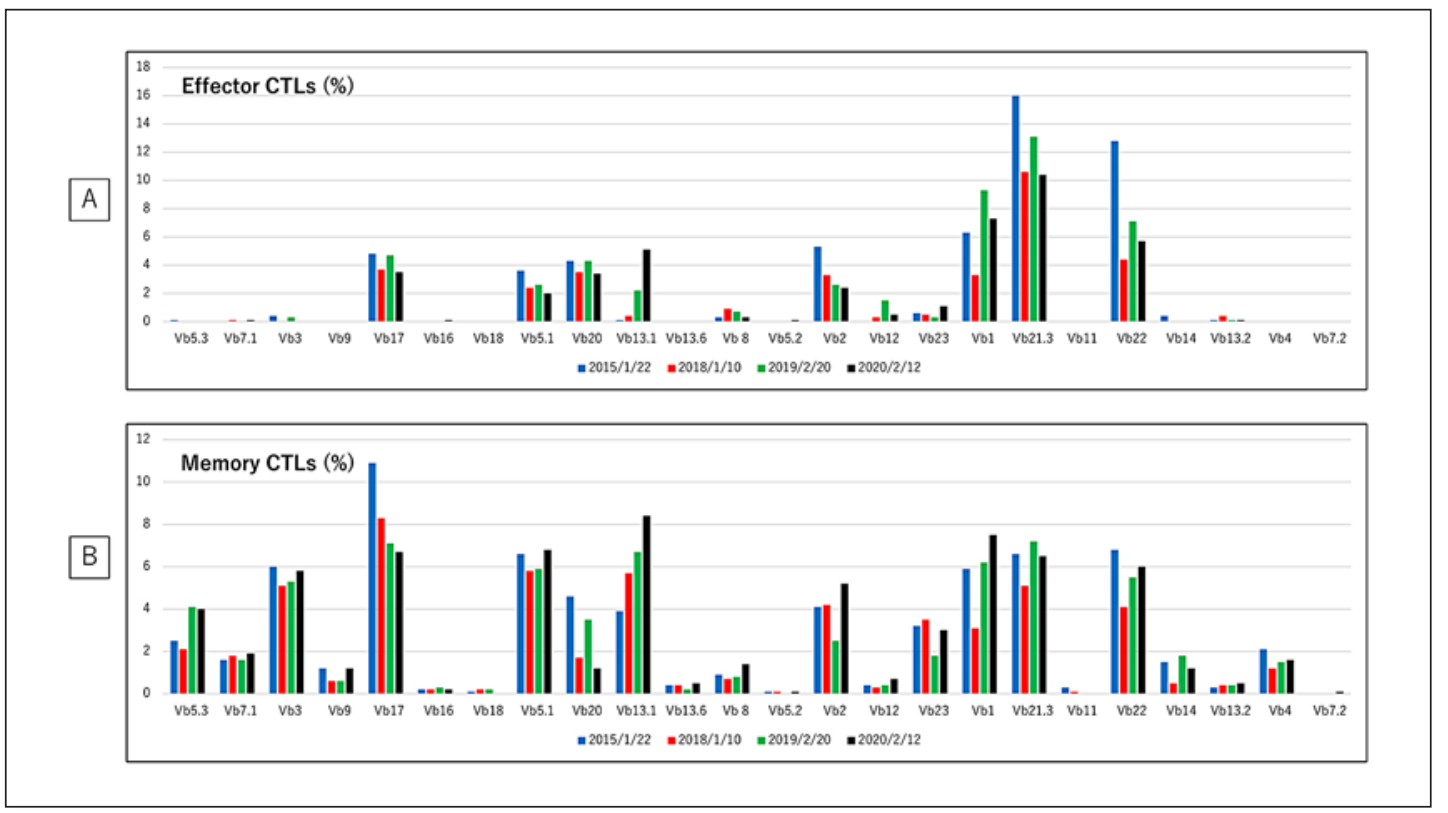

Fig. 3. T-cell receptor repertoire analyses. T-cell receptor repertoire analyses were performed using IOTest $V$ beta gene repertoire kit (Beckman Coulter, Tokyo, Japan) in January 2015 (blue bar), January 2018 (red bar), February 2019 (green bar), and February 2020 (black bar). A Effector CTLs (\%). B Memory CTLs (\%). $\mathrm{Vb}, \mathrm{V}$ beta gene.

was observed. Furthermore, the number of CTLs expressing the TCR $V$ beta 13.1 has been gradually increasing since the occurrence of HCC.

Written informed permission for use and disclosure of the protected health information was obtained from the patient.

\section{Discussion/Conclusion}

ATLL is one of the refractory neoplasms to treat [4], and relapse of ATLL means that it is nearly impossible to achieve remission. However, this patient attained long-term survival with CR for $>9$ years. Conversely, HTLV-1 was not eliminated; approximately 25-65 copies of HTLV-1 provirus/1,000 peripheral mononuclear cells are detected to date. The maintenance and circulation of Tax-specific CTLs may have played a role in preventing the relapse of ATLL. Pegylated interferon alpha-2b may have also induced and activated Tax-specific CTLs.

The cause of HCC development after obtaining sustained virological response with DAA treatment is currently unknown [5]. One possible cause of HCC pathogenesis is an immunological mechanism [6]. Sustained HCV infection causes chronic inflammation in liver cells by triggering an immune response. A swift clearance of HCV by DAA may prevent an immune response and lead to the development of HCC. HCV-specific CTLs have been detected and maintained in this patient. The remnant cells infected with HCV may have developed into HCC cells and were subsequently eradicated by the HCV-specific CTLs. Another hypothesis is that CTLs specific for HCC but unrelated HCV antigens, such as the CTLs expressing TCR $V$ beta 13.1, may have risen and killed the HCC after transient immunological suppression by the DAA treatment. Strong anticancer immunological antitumor activity that overcame ATLL relapse may have contributed to the eradication of HCC in this patient. 
In summary, herein, we report an impressive case of a patient who achieved and maintained CR for ATLL and HCC, most likely owing to CTLs. Tax- and HCV-specific CTLs may have been induced because their genome sequences do not resemble any sequences in the human genome.

\section{Acknowledgment}

The authors thank Ms. Riyo Matsumoto for editing the manuscript.

\section{Statement of Ethics}

Written informed consent to publish the content of the manuscript was obtained from the patient concerned.

\section{Conflict of Interest Statement}

The authors have no conflicts of interest to declare.

\section{Funding Sources}

There was no funding to prepare the manuscript.

\section{Author Contributions}

T.J. designed the work and acquired, analyzed, and interpreted data, as well as wrote the manuscript. Y.K., K.M., H.S., T.S., H.T., and S.H. conducted the acquisition and the analysis of data. T.O., M.M., and J.T. discussed the conception of the manuscript.

\section{References}

1 Rossjohn J, Gras S, Miles JJ, Turner SJ, Godfrey DI, McCluskey J. T cell antigen receptor recognition of antigenpresenting molecules. Annu Rev Immunol. 2015;33:169-200.

2 Tsukasaki K, Utsunomiya A, Fukuda H, Shibata T, Fukushima T, Takatsuka Y, et al. VCAP-AMP-VECP compared with biweekly CHOP for adult T-cell leukemia-lymphoma: Japan Clinical Oncology Group Study JCOG9801. J Clin Oncol. 2007 Dec;25(34):5458-64.

3 Wilson WH, Bryant G, Bates S, Fojo A, Wittes RE, Steinberg SM, et al. EPOCH chemotherapy: toxicity and efficacy in relapsed and refractory non-Hodgkin's lymphoma. J Clin Oncol. 1993 Aug;11(8):1573-82.

4 Ishitsuka K, Tamura K. Human T-cell leukaemia virus type I and adult T-cell leukaemia-lymphoma. Lancet Oncol. 2014 Oct;15(11):e517-26.

5 Nault JC, Colombo M. Hepatocellular carcinoma and direct acting antiviral treatments: controversy after the revolution. J Hepatol. 2016 Oct;65(4):663-5.

6 Kogiso T, Sagawa T, Kodama K, Taniai M, Katagiri S, Egawa H, et al. Hepatocellular carcinoma after directacting antiviral drug treatment in patients with hepatitis C virus. JGH Open. 2018 Nov;3(1):52-60. 